\title{
Percepção e vivência dos enfermeiros acerca dos cuidados paliativos no contexto
}

\section{hospitalar}

\author{
Perception and experience of nurses about palliative care in the hospital context \\ Percepción y experiencia de enfermeras sobre cuidados paliativos en el contexto hospitalario
}

Recebido: 20/03/2021 | Revisado: 29/03/2021 | Aceito: 05/04/2021 | Publicado: 12/04/2021

Ana Paula Garcia de Almeida

ORCID: https://orcid.org/0000-0002-8265-4678 Universidade Franciscana, Brasil

E-mail: anapaulaalmeida845@gmail.com

Claudia Zamberlan

ORCID: https://orcid.org/0000-0003-1898-328X Universidade Franciscana, Brasil

E-mail: claudiaz@ufn.edu.br

Cristina de Freitas Rodrigues

ORCID: https://orcid.org/0000-0001-7246-8882 Universidade Franciscana, Brasil

E-mail: cristina.rodrigues@ufn.edu.br

Silomar Ilha

ORCID: https://orcid.org/0000-0002-2132-9505 Universidade Franciscana, Brasil

E-mail: silomar.ilha@ufn.edu.br

\begin{abstract}
Resumo
Objetivou-se conhecer a percepção e vivência dos enfermeiros acerca dos cuidados paliativos no contexto hospitalar. Trata-se de um estudo exploratório e descritivo de abordagem qualitativa. Fizeram parte do estudo enfermeiros(as) e enfermeiras residentes de um hospital público do estado do Rio Grande do Sul, totalizando nove profissionais de uma unidade clínica. Os dados foram coletados por meio de entrevistas semiestruturadas ao longo do primeiro semestre de 2020 e foram analisados com base na técnica de análise de conteúdo. Da análise dos dados emergiu duas categorias: Cuidados Paliativos - temática complexa; e Vivências heterogêneas acerca dos cuidados paliativos. Na primeira categoria percebe-se que falar de cuidados paliativos é abordar uma temática complexa com diferentes enfoques. Na segunda, foram relatadas experiências diversas relacionadas ao tema, evidenciando que, apesar de ser uma temática pouco refletida e discutida faz parte da rotina de muitos profissionais e famílias. Conclui-se que apesar de uma visão limitada sobre cuidados paliativos da maioria dos profissionais, a temática é complexa por delinear aspectos biopsicossocioespirituais das pessoas em situação de terminalidade e que as vivências acontecem de formas heterogêneas. Além disso, os depoimentos foram relevantes para demonstrar que há fragilidade em relação à percepção dos cuidados paliativos, o que remete a um possível despreparo do tema.
\end{abstract}

Palavras-chave: Cuidados paliativos; Enfermeiros; Hospital.

\begin{abstract}
The objective was to know the nurses' perception and experience about palliative care in the hospital context. This is an exploratory and descriptive study with a qualitative approach. The study included nurses and nurses residing in a public hospital in the state of Rio Grande do Sul, totaling nine professionals from the clinical unit. Data were collected through semi-structured interviews in August 2020. Through the analysis of the data two categories emerged, namely: Palliative Care - complex theme, and Heterogeneous experiences about palliative care. In the first category, it is clear that talking about palliative care is to address a complex theme with different approaches. In the second category, several experiences were reported, showing that, despite being a theme that is still discussed, it ends up being part of the routine of many professionals and families. It is concluded that despite the limited view on palliative care of most professionals, the theme is complex because it outlines biopsychosocio-spiritual aspects of people in terminal situations, in particular, and that the experiences happen in heterogeneous ways. In addition, the statements were relevant to demonstrate that there is weakness in relation to the perception of palliative care, which refers to a possible unpreparedness of the topic.
\end{abstract}

Keywords: Palliative care; Nurse; Hospital.

\section{Resumen}

El objetivo fue conocer la percepción y experiencia de las enfermeras sobre los cuidados paliativos en el contexto hospitalario. Se trata de un estudio exploratorio y descriptivo con enfoque cualitativo. El estudio incluyó a enfermeras 
y enfermeras residentes en un hospital público del estado de Rio Grande do Sul, totalizando nueve profesionales de una unidad clínica. Los datos se recolectaron a través de entrevistas semiestructuradas durante el primer semestre de 2020 y se analizaron con base en la técnica de análisis de contenido. Surgieron dos categorías del análisis de datos: cuidados paliativos - tema complejo y experiencias heterogéneas sobre cuidados paliativos. En la primera categoría, está claro que hablar de cuidados paliativos es abordar un tema complejo con diferentes enfoques. En la segunda, se reportaron diversas experiencias relacionadas con el tema, mostrando que, a pesar de ser un tema mal reflejado y discutido, termina siendo parte de la rutina de muchos profesionales y familias. Se concluye que a pesar de la visión limitada sobre cuidados paliativos de la mayoría de los profesionales, el tema es complejo porque perfila aspectos biopsicosocio-espirituales de personas en situaciones terminales, en particular, y que las vivencias suceden de manera heterogénea. Además, los enunciados resultaron relevantes para demostrar que existe debilidad en relación a la percepción de los cuidados paliativos, lo que hace referencia a una posible falta de preparación del tema.

Palabras clave: Cuidados paliativos; Enfermeras; Hospital.

\section{Introdução}

A terminalidade, refletida no sentido das fases finais de vida e prévia à morte do ser humano, é temática relevante para o profissional da saúde, familiares e amigos da pessoa assistida. O processo de terminalidade da vida de um indivíduo, em que está envolvida uma equipe de profissionais de saúde, demanda enfrentamento de numerosos problemas, sentimentos e sintomas, os quais se configuram como intensos e que representam significativo impacto emocional para o paciente, seus familiares, bem como, à equipe de saúde que o auxilia (Academia Nacional De Cuidados Paliativos [ANCP], 2012).

A denominação "cuidados paliativos" é utilizada para designar a ação de uma equipe multiprofissional à pacientes fora de possibilidades terapêuticas de cura. A palavra "paliativa" origina-se do latim palliun que significa manto e proteção, indicando que seu significado mais profundo é o da proteção em relação aos que já não têm mais oportunidades de cura (Silva et al, 2012; Andrade et al, 2016). A Organização Mundial de Saúde (OMS) definiu em 1990 e redefiniu em 2002 que os Cuidados Paliativos tratam do aprimoramento da qualidade de vida, dos pacientes e famílias que enfrentam problemas associados com doenças, por meio da prevenção e do alívio do sofrimento, corroborados pela identificação precoce, avaliação correta, tratamento da dor e outros problemas de ordem física, psicossocial e espiritual, também no momento da morte (Rabow et al, 2010; Conceição et al, 2019).

Essa definição reafirma a importância da vida, considerando a morte como um processo natural, assim como, no estabelecimento de um cuidado que não acelere a chegada da morte, nem a prolongue com medidas desproporcionais (obstinação terapêutica), além de possibilitar alívio da dor e de outros sintomas, integrar os aspectos psicológicos e espirituais na estratégia do cuidado e oferecer um sistema de apoio à família para que possam enfrentar a doença do paciente e o período de luto (Silva et al, 2012; Andrade et al, 2016).

A fase final da vida ocorre permeada por sentimentos dolorosos e, por vezes, conflituosos, quando se fala em cuidados paliativos. Assim, a paliação é fundamental e complexa o suficiente para viabilizar um cuidado e atenção contínua e específica não somente ao paciente, mas à sua família. O caráter assistencial em cuidados paliativos pode contribuir para a humanização do processo de morrer, sendo que esta modalidade de cuidado pode ser oferecida em instituições de saúde, bem como na própria residência (Silva \& Moreira 2011).

De acordo com estudos do Instituto Brasileiro de Geografia e Estatística (IBGE), a expectativa de vida dos brasileiros aumentou e os profissionais de saúde começaram a perceber que mesmo não havendo cura em diversas situações, há uma possibilidade de atendimento com ênfase na qualidade de vida e cuidados aos pacientes. Essa forma de atendimento, ocorre por meio de uma assistência interdisciplinar e da abordagem que contemple os familiares no compartilhamento do processo de cuidado, uma vez que os mesmos participam do momento final da vida dessas pessoas (Hermes \& Lamarca, 2013).

A assistência oferecida por uma equipe multiprofissional durante o processo de terminalidade em instituições hospitalares se revela um tema de grande relevância. Por essa razão, merece ser pesquisado e estudado, no intuito de melhorias e benefícios para todos, visto que grande parte da população em virtude das dificuldades socioeconômicas, busca a 
hospitalização. Sendo assim, a equipe que assiste o paciente em terminalidade no cenário hospitalar, merece obter respaldo ético e legal, afim de que se desenvolvam cada vez mais possibilidades de oferecer cuidados efetivos, qualificando a assistência, valorizando a integralidade e a dignidade humana (ANCP, 2012).

Neste enfoque, o estudo se justifica por contemplar um tema emergente que está presente no cotidiano do fazer profissional do enfermeiro e, para tanto, precisa ser investigado e refletivo por esta categoria profissional. Com base nestas considerações e com destaque ao tema no contexto atual, assim como, no processo de trabalho do profissional enfermeiro temse como questão norteadora deste estudo: Como o enfermeiro percebe e vivência os cuidados paliativos no contexto hospitalar? Assim, a fim de ampliar a discussão sobre a temática proposta, o estudo teve por objetivo conhecer a percepção e vivência dos enfermeiros acerca dos cuidados paliativos no contexto hospitalar.

\section{Metodologia}

Trata-se de uma pesquisa de cunho exploratório e descritivo de abordagem qualitativa, desenvolvida com enfermeiros(as) atuantes em um hospital público do estado do Rio Grande do Sul e enfermeiras residentes com atividades práticas na mesma instituição. A pesquisa qualitativa considera as representações sociais que abarcam a experiência das relações objetivas entre os atores sociais e os significados por estes impostos e também a dinâmica das relações que estabelecem a construção do conhecimento exterior ao sujeito. Esse método se faz pertinente por desenvolver de maneira detalhada o caráter social e as dificuldades acerca da construção de conhecimento que o abrangem de forma parcial e incompleta (Minayo, 2008). O hospital, onde desenvolveu-se o estudo conta com 112 leitos, e, caracteriza-se por ser um hospital público de médio porte, com 100\% dos atendimentos pelo Sistema Único de Saúde (SUS) e referência para atendimentos da região de Verdes Campos e Entre Rios associados a $4^{\text {a }}$ Coordenadoria Regional de Saúde.

Considerou-se como critérios de inclusão do estudo, ser enfermeiro(a) ou enfermeira residente do cenário do estudo, com atuação mínima de seis meses no referido hospital e com atuação nos três turnos de trabalho, quais sejam: manhã, tarde e noite. Atenderam aos critérios e compuseram o corpus dessa pesquisa nove profissionais.

Os dados foram coletados no período de agosto de 2020 por meio de uma entrevista semiestruturada e individual, em dia e horário previamente agendado com os participantes em um local privativo e sem a interferência de outras pessoas. As entrevistas tiveram uma média de 10 minutos e seguiram um roteiro previamente definido, constituído pelos dados sócio demográficas das (os) participantes e pelos dados específicos acerca da percepção e vivência com cuidados paliativos. Todas as entrevistas foram gravadas em um celular da marca Samsung e, posteriormente, transcritas pela pesquisadora principal.

Os dados sócios demográficos foram analisados de modo descritivo considerando as seguintes variáveis: idade, sexo, religião, nível de formação, tempo de formação, tempo de atuação na área, tempo de atuação na instituição. A segunda parte do estudo foi analisada por meio da análise de conteúdo. De acordo com Bardin (2011), esta análise propõe as seguintes etapas: pré-análise, exploração do material, categorização e o tratamento dos resultados.

Na primeira etapa, pré-análise, os pesquisadores organizaram o material estabelecendo a leitura dos dados elencados pelos participantes por meio das unidades de análise. A segunda etapa, definida como a exploração do material, possibilitou que os pesquisadores, aplicassem, de forma organizada, as decisões tomadas, a definição das categorias, classificando os elementos constitutivos de um conjunto caracterizados por diferenciação e realizando o reagrupamento por analogia por meio de critérios definidos previamente no sentido de propiciar a realização da inferência. Por fim, na terceira etapa, ocorreu o tratamento dos resultados obtidos e sua interpretação (Bardin, 2011).

Consideraram-se os preceitos éticos e legais, conforme a Resolução 466/12 do Ministério da Saúde (Brasil, 2012). O Projeto foi aprovado por Comitê de Ética em Pesquisa sob o Parecer nº 4.192.328 e CAAE: 34986820.7.0000.5306. Manteve- 
se o anonimato dos participantes, os identificando ao longo do texto, por nomes de flores escolhidas pelos mesmos no momento da coleta dos dados.

\section{Resultados e Discussão}

Dos nove Profissionais participantes do estudo, dois eram do sexo masculino e sete do feminino, predominantemente católicos, com idades entre 24 e 44 anos. Em relação ao tempo de formação, a maior prevalência foi de dois a quatro anos, sendo apenas 33\% deles com tempo de formação superior a cinco anos. Os dados analisados, resultaram em duas categorias, quais sejam: Cuidados Paliativos - temática complexa; e Vivências heterogêneas acerca dos cuidados paliativos.

\subsection{Cuidados Paliativos - temática complexa}

A primeira categoria que emergiu acerca dos dados das entrevistas diz respeito a percepção dos enfermeiros sobre cuidados paliativos e permitiu a percepção de que falar de cuidados paliativos é abordar uma temática complexa com diferentes enfoques. Assim, quando questionados sobre suas percepções acerca da temática, alguns descreveram como aquela assistência prestada a condições em que não há mais possibilidade de cura, apenas o controle dos sintomas, do sofrimento, conforme seguem depoimentos:

"[...] eu entendo como tu tratar o sofrimento do paciente. Não é se voltar pra doença em específico, mas sim questão de cuidar daquele sofrimento que aquela doença está causando. Aborda a questão de dor, a questão espiritual do paciente, a questão social dele, cuida o sofrimento dele." (Lírio)

“[...] é proporcionar para o paciente um conforto [...]” (Rosa)

"Cuidados paliativos eu acho que é agente oferecer um maior conforto para um paciente que não tenha um bom prognóstico.”(Violeta)

"É um cuidado que vai ser prestado para um paciente que não tem como reverter à situação dele, é uma patologia que não tem cura, que ele vai conviver com ela até os últimos dias da vida dele. Tu vai manter aquele paciente confortável, vai aliviar a dor, vai manter o paciente limpo, com uma dignidade, mas não vai trazer muita perspectiva de melhora e não vai ter cura." (Girassol)

Pode-se observar nos depoimentos que, para os enfermeiros dessa unidade de internação clínica, os cuidados paliativos, de modo geral, são aqueles que amenizam a dor e proporcionam conforto ao paciente em fase terminal da vida. De acordo com a OMS, os cuidados paliativos surgiram como uma nova modalidade de cuidado, que tem como preceito a melhora da qualidade de vida de pacientes e seus familiares diante do enfrentamento de doenças que ameaçam a vida, em sua maioria sem perspectiva de cura, por meio da prevenção e alívio dos sofrimentos físicos, psicossociais e espirituais (Organização Mundial da Saúde [OMS], 2002). Sendo assim, os depoimentos vão ao encontro da definição de paliar, destacando em especial a preocupação dos profissionais com o alívio dos sintomas álgicos e do sofrimento psicossocial que o agravo pode causar.

Além disso, pode-se perceber a inclusão da família no processo de cuidado e no acolhimento, a preocupação com a humanização, corroborando com o que é preconizado pela OMS.

"[...] os cuidados paliativos envolvem todo o ser, precisa muito de humanização em primeiro lugar. É você estar com o outro, saber lidar com a dor do outro, sabendo se colocar naquela situação com o outro. Tanto com o paciente, quanto com um familiar que está ali com uma fragilidade muito grande." (Jasmin) 
Com a fala acima, ressalta-se a importância de uma assistência com olhar humanizado e integrado por meio da sensibilização e orientação dos profissionais envolvidos nessa dinâmica de saúde desafiadora e necessária. Assim, o termo humanização tem o intuito de cuidado e valorização da pessoa humana, da relação interpessoal que deve ser firmada entre paciente, profissional e família, ao desenvolver um cuidado de forma afetuosa ao próximo (Palmeira, Comim, Peres, 2011). Nos cuidados paliativos, a equipe de saúde, paciente e família criam um vínculo de confiança e, em conjunto, estabelecem um plano estratégico de assistência integral e contínua, onde o paciente é agente ativo no tratamento.

Vale ressaltar que os cuidados paliativos podem e devem ser oferecidos em associação aos cuidados curativos, pois contribuem para a prevenção e tratamento do sofrimento do paciente e seus familiares. Enquanto houver vida, sempre existirá a necessidade do cuidado de enfermagem e sempre haverá muito que se fazer para a pessoa vivenciar os últimos dias de sua existência sem sofrimento (Silva et al, 2010).

Contudo, percebe-se nas falas, uma visão limitada sobre o conceito de cuidado paliativo, os quais não deveriam ser uma opção para o paciente somente quando os tratamentos tradicionais se esgotarem. Há muito a se fazer em conjunto com as terapêuticas clássicas de forma paliativa complementar. Quase sempre é possível ampliar os tratamentos modificadores da doença e melhorar o cuidado para o paciente. Através da atuação multiprofissional especializada em cuidados paliativos o paciente se beneficia e desenvolve um melhor entendimento dos impactos de sua doença, aceitação e planejamento de vida. $\mathrm{O}$ Cuidado Paliativo não se trata de "desistir" ou "investir", se trata de zelar pelo conforto e bem-estar do paciente, de forma complementar a terapêutica tradicional.

\subsection{Vivências heterogêneas acerca dos cuidados paliativos}

Quando questionados sobre vivências com a temática desse estudo, foram relatadas experiências com pacientes crônicos críticos, com pacientes oncológicos e até mesmo com seus próprios familiares. Este fato evidencia que, apesar de ser uma temática que ainda pouco discutida entre os profissionais, por motivos diversos tais como: medo, negação e até mesmo desconhecimento, é tema que faz parte da rotina de muitos profissionais e famílias.

"Muitas vivências, eu trabalhei dois anos em outra cidade e eu lidei com todos os tipos de casos assim, do início ao fim da vida." (Jasmin)

"[...] a gente tem alguns pacientes, os quais discutimos ali e daí tu vê que daqui a pouco ele entrou pra medidas de conforto, pra cuidado paliativo." (Tulipa)

“Tive aqui na instituição, tive em casa também lógico, né, família [...]”( Orquídea)

"Eu faço tratamento oncológico de pacientes que estão com câncer, que já iniciam um paliativo, ou aqueles que no decorrer do tratamento se torna um paliativo. Aquele paciente que era curativo [...] e a doença progrediu e daí por diante se faz um tratamento paliativo." (Cravo)

Diferentemente do que foi relatado pelos profissionais, de modo geral a literatura nos traz relatos de vivências homogêneas, relacionadas à pacientes terminais de tratamento oncológico, justificando mais uma vez a importância de estudos que abordem essa temática. Inicialmente, o cuidado paliativo era oferecido aos indivíduos com câncer, entretanto, ao mesmo tempo em que esse tipo de cuidado ganhou prestígio, cresceu o interesse em oferecê-lo a indivíduos com outras doenças como as doenças neurodegenerativas e insuficiências orgânicas (Cardoso et al, 2013).

Em relação à área oncológica destaca-se que de modo geral o diagnóstico do câncer acontece tardiamente, impossibilitando tratamento curativo, pois a maioria dos indivíduos apresenta doença avançada, o que acaba impactando de forma negativa sobre o indivíduo e seus familiares. Sob esse enfoque, os cuidados paliativos têm grande importância no controle dos sofrimentos físico, espiritual e psicossocial (Freitas \& Pereira, 2013). Entretanto, independentemente do cenário 
de vivência do cuidado paliativo, algumas situações e sentimentos serão análogos. Falar sobre a efemeridade da vida nem sempre é um assunto fácil, mesmo que isso seja parte do ciclo da vida, pois a morte é um fenômeno que acarreta profundas reações emocionais, conforme demonstram as falas abaixo:

"O sentimento em si da gente é tipo uma impotência, é que a gente sabe que aquilo ali é uma coisa que é fisiológica da pessoa que já não tá mais funcionando 100\%, é uma fase bem difícil. Eu acho que as pessoas que tem um familiar nessa condição tem que ter um emocional muito seguro, tem que ser um emocional muito firme [...] pra mim foi muito difícil de eu trabalhar na clínica, eu tive que me blindar, porque eu sou uma pessoa que por qualquer coisinha eu choro com o paciente, eu choro com o familiar, e eu falo nisso e eu encho os olhos de água, não tem como não, a gente tem que admitir que somos humanos, a gente não pode ser como todo mundo é, "ah vai morrer e deu", "vai morrer e pronto!". (Azaleia)

"[...] Para mim mexeu muito comigo e me fortaleceu dentro da saúde, na minha profissão, faz eu gostar mais da minha profissão, pessoas de todas as idades a gente vê no final da vida, pessoas da minha idade." (Jasmin)

Ao falar sobre os aspectos relacionados ao cotidiano de trabalho, que envolvem a finitude da vida, percebe-se que por vezes, alguns profissionais encontraram dificuldades no processo de aceitação. Algumas vivências foram marcadas por sofrimento e angústia, atrelados ao fato de se sentirem impotentes, frustrados com relação à morte e solidários diante do paciente e família. O sentimento de impotência deve-se principalmente ao fato de que os profissionais da saúde são preparados para salvar, preservar e prolongar a vida (Cardoso et al, 2013; Vicensi, 2016).

Os profissionais de saúde tendem a passar grande parte do seu tempo no desenvolvimento de cuidados diretos a pacientes e familiares e, ao se deparar com situações do processo de terminalidade, compartilham suas angústias e dificuldades, sendo um importante momento de trocas em suas vidas (Cardoso et al, 2013). Assim, os dados desse estudo demonstram que a vivência dos enfermeiros em relação aos cuidados paliativos, apontam para o cuidado de formas diversas, sendo sua vivência construída e reconstruída ao longo de sua vida pessoal e profissional.

\section{Considerações Finais}

Essa pesquisa atendeu ao seu objetivo de conhecer a percepção e vivência dos enfermeiros acerca dos cuidados paliativos no contexto hospitalar. Assim, destaca-se que além de identificar a percepção dos enfermeiros foi possível refletir sobre a importância dos cuidados paliativos no contexto estudado.

Os dados demonstraram que os cuidados paliativos são vivenciados como uma temática complexa por delinear aspectos biopsicosocioespirituais das pessoas em situação de terminalidade e que as vivências acontecem de formas heterogêneas. Além disso, os depoimentos foram relevantes para demonstrar que há fragilidade em relação à percepção dos cuidados paliativos, o que remete a um possível despreparo acerca do tema e tudo que ele envolve.

Como limitações dessa pesquisa, encontra-se o fato da mesma ter sido realizada em somente um hospital, em apenas uma unidade assistencial. Assim, torna-se necessário que novos estudos sejam realizados para identificar melhor a percepção dos enfermeiros sobre cuidados paliativos em diferentes contextos e cenários. Sendo assim, reafirma-se a importância de desenvolver estudos sobre a percepção da temática, a fim de delinear as estratégias para um cuidado integral na terminalidade. 


\section{Referências}

Academia Nacional De Cuidados Paliativos [ANCP] (2012). Critérios de Qualidade para Unidades de Cuidados Paliativos. Associação Nacional de Cuidados Paliativos, 2012. 590p.

Andrade, C. G., et al. (2016). Cuidados paliativos e bioética: estudo com enfermeiros assistenciais. Rev. Cuidado é fundamental, 8(4), $4922-28$.

Andrade, C. G., Costa, S. F. G., \& Lopes, M. E. L. (2013). Cuidados paliativos: a comunicação como estratégia de cuidado para o paciente em fase terminal. Ciência \& Saúde Coletiva, 18(9), 2523-30.

Brasil. Conselho Nacional de Saúde. (2012). Diretrizes e normas regulamentadoras de pesquisa em seres humanos. Resolução ${ }^{\circ}{ }^{466}$, de 12 de dezembro de 2012 .

Brasil. (2018). Agenda de Prioridades de Pesquisa do Ministério da Saúde - APPMS/ Ministério da Saúde, Secretaria de Ciência, Tecnologia e Insumos Estratégicos, Departamento de Ciência e Tecnologia. - Brasília: Ministério da Saúde.

Bardin, L. (2012) Análise de Conteúdo.São Paulo: Edições 70.

Cardoso, D. H. et al. (2013). Cuidados paliativos na assistência hospitalar: a vivência de uma equipe multiprofissional. Texto contexto - enferm., 22(4),113441.

Carvalho, K. K., et al. (2017). Processo educativo em cuidados paliativos e a reforma do pensamento. Rev. Invest. educ. enferm., 35(1), 17-25.

Conceição, M. V., et al. (2019). Conhecimento sobre cuidados paliativos entre médicos residentes de hospital universitário. Revista Bioética, $27(1), 134-42$.

Fernandes, M. A., et. al. (2013). Percepção dos enfermeiros sobre o significado dos cuidados paliativos em pacientes com câncer terminal. Ciência \& Saúde Coletiva, 18(9), 2589- 96

Freitas, N. O., \& Pereira, M. V. G. Percepção dos enfermeiros sobre cuidados paliativos e o manejo da dorna UTI. O Mundo da Saúde, $37(4), 450-457$.

Hermes, H. R., \& Lamarca, I. C. A. (2013) Cuidados paliativos: uma abordagem a partir das categorias profissionais de saúde. Ciência \& Saúde Coletiva, $18(9), 2577-88$.

Instituto Brasileiro de Geografia e Estatística [IBGE]. (2006). Estatísticas do Século XX. Brasil, Rio de Janeiro.

Lakatos, E. M., \& Marconi, M. A. (2017). Técnicas de Pesquisa. São Paulo: Atlas.

Minayo, M. C (2008). O desafio do conhecimento: pesquisa qualitativa em saúde. 11. ed. São Paulo: Hucitec.

Minayo, M. C. S (ORG). (2011). Pesquisa Social: Teoria, Método e Criatividade. Petrópolis: ed Vozes.

Morais, E. N., et. al. (2018). Cuidados paliativos: enfrentamento dos enfermeiros de um hospital privado na cidade do Rio de Janeiro-RJ. Rev.Cuidado é Fundamental, 10(2), 318-325.

Organização Mundial de Saúde [OMS]. (2002). National cancer control programmes: policies and managerial guidelines. Geneva: WHO.

Palmeira, H. M., et al. (2011). Cuidados paliativos no Brasil: revisão integrativa da literatura científica. Aletheia, (35-36), 179-189.

Rabow, M.W., et. al. (2010). The intersection of need and opportunity: assessing and capitalizing on opportunities to expand hospital-based palliative care services. J Palliat Med., 13(10), 1205-10.

Santos, B. C., et. al. (2017). A percepção dos enfermeiros de um hospital geral sobre os cuidados paliativos. Rev de Enfermagem, 11(6), 2288-93.

Silva, H. A., et. al. (2018). Intervenção em cuidados paliativos: conhecimento e percepção dos enfermeiros. Rev de Enfermagem,12(5), 1325-30.

Silva, M. M., et. al. (2012). Análise do cuidado de enfermagem e da participação dos familiares na atenção paliativa oncológica. Texto Contexto Enferm, $21(3), 658-66$.

Silva, M. M., \& Moreira, M. C. (2011). Sistematização da assistência de enfermagem em cuidados paliativos na oncologia: visão dos enfermeiros.Rev.Acta Paulista de Enfermagem, 24(2),172-78.

Silva, C. C. B., et al. (2010). Análise da percepção do enfermeiro sobre a assistência de enfermagem ao paciente em cuidados paliativos. Revista Nursing, 13(146), 411-6.

Vega, M. E. P., \& Cibanal, L. J. (2016). Impacto psicosocial en enfermeras que brindan cuidados en fase terminal. Revista Cuidarte,7(1), 1210-18.

Vicensi, M. C. (2016). Reflexão sobre a morte e o morrer na UTI: a perspectiva do profissional. Rev. Bioét., 24(1). 